Bull. Austral. Math. Soc.

VOL. 37 (1988) [389-410]

\title{
THE DIRICHLET PROBLEM FOR DEGENERATE ELLIPTIC 2-DIMENSIONAL MONGE-AMPERE EQUATION
}

\author{
KAZUO AMANO
}

\begin{abstract}
We study the following Dirichlet problem for the degenerate elliptic Monge-Ampère equation: Given $f \in C^{\prime \cdot}(\bar{\Omega}), f \geq 0$ and $g \in C^{:+2}(\bar{\Omega})$, find a solution $u \in C^{t}(\bar{\Omega}), t \geq 2$, satisfying $u_{11} u_{22}-u_{12}^{2}=f$ in $\Omega$ and $u=g$ on $\partial \Omega$. Since $f$ is nonnegative, we cannot. apply any standard elliptic methods. In this paper, we use an iteration scheme of NashMoser type and a priori estimates for degenerate elliptic operators, and solve the Dirichlet problem for a certain class of $f$ and $g$.
\end{abstract}

\section{INTRODUCTION}

There are vast references on elliptic Monge-Ampère equations (see [16]). However, we find only a few papers concerned with degenerate elliptic Monge-Ampère equations. Bakelman [3] and Rauch-Taylor [14] studied existence and uniqueness of weak solutions. Lin [10] proved $C^{\prime 3}$ local solvability. Trudinger [15] and C'hen [5] solved boundary value problems in the function spaces $C^{1,1}$ and $W^{2, \infty}$ respectively. Unfortunately, no one lhas invest.jgated the Dirichlet problem and its loss of clerivatives in $C^{\text {s }}$ classes. In this paper we shall solve the following problem for a certain class of $f$ and $g$.

Problem. Given $f \in C^{* *}(\bar{\Omega}), f \geqslant 0$ and $g \in C^{s *+2}(\bar{\Omega})$, find a solution $u \in$ $(\because \cdot(\bar{\Omega})$ satisfying

$$
u_{11} u_{22}-u_{12}^{2}=f \quad \text { in } \quad \Omega, \quad u=g \quad \text { on } \quad \partial \Omega
$$

Here $\Omega$ is a bounded open set in $\mathbb{R}^{2}$ with $C^{\infty}$ boundary, $s_{*}$ is a nonnegative integer and $t_{*}$ is a function of $s_{*}$ satisfying $t_{*} \rightarrow \infty$ as $s_{*} \rightarrow \infty, u_{i j}$ denotes the derivative $\partial_{i} \partial_{j} u$.

All the difficulties of the problem arise from the nonnegativity of $f$, In fact, since $f \geqslant 0$, standard elliptic methods (see $[4],[7-\theta],[11-12]$ ) do not work for highes derivative estimates. Furthermore, the following two examples (Examples 1.1 and 1.2) show that we cannot always find a solution $u$ of the problem for general $f$ and $g$; this implies that we should place some restriction on $f$ and $g$.

In order to illustrate the difficulties of the problem, we consider a radially symmetric case and seek exact solutions. If $\Omega=B=\{r: r<1\}, f=f(r), g=g(r)$ and

Received 27 July 1987

Copyright Clearance Centre, lnc. Serial-fee code: 0004-9ז29/88 \$A2.00+0.00. 
$u=u(r)$, where $r=\sqrt{x_{1}^{2}+x_{2}^{2}}$, then we can transform (1.1) into an ordinary differential equation

$$
\left(u_{r}^{2}\right)_{r}=2 r f \quad \text { in } B, \quad u=g \text { on } \partial B .
$$

It is easy to solve (1.1)'. In fact, direct computation gives

$$
u=\int_{1}^{r}\left[2 \int_{0}^{s} t f(t) d t\right]^{1 / 2} d s+g(1)
$$

Hence, we have the following

EXAMPLE 1.1: For $f=r^{\nu}, \nu=1,2, \ldots$

$$
u=\frac{2 \sqrt{2}}{(\nu+4) \sqrt{\nu+2}}\left(r^{\frac{\nu}{2}+2}-1\right)+g(1)
$$

is a solution of (1.1)'. If $\nu$ is even and $\nu / 2$ is odd, then

$$
f \in C^{+\infty}(\bar{B}) \text { and } u \notin C^{\frac{\nu}{2}+2}(\bar{B}) \text {. }
$$

EXAMPLE 1.2: For $f=\left|r-\frac{1}{2}\right|^{\nu}, \nu=1,2, \ldots$, we have

$$
u_{r}= \begin{cases}\left.-\frac{1}{(\nu+1)(\nu+2)}\{2(\nu+1) r+1\}\left[\frac{1}{2}-r\right]^{\nu+1}+\frac{1}{(\nu+1)(\nu+2)}\left[\frac{1}{2}\right]^{\nu+1}\right]^{\frac{1}{2}} & \text { if } 0 \leqslant r \leqslant \frac{1}{2} \\ {\left[\frac{1}{(\nu+1)(\nu+2)}\{2(\nu+1) r+1\}\left[r-\frac{1}{2}\right]^{\nu+1}+\frac{1}{(\nu+1)(\nu+2)}\left[\frac{1}{2}\right]^{\nu+1}\right]^{\frac{1}{2}}} & \text { if } \frac{1}{2} \leqslant r \leqslant 1 .\end{cases}
$$

This implies

$$
u \in C^{1}(\bar{B}) \text { and } u \notin C^{2}(\bar{B}) \text {. }
$$

Examples 1.1 and 1.2 show that in the problem the function $t_{*}=t_{*}\left(s_{*}\right)$, which tends to $\infty$ as $s_{*} \rightarrow \infty$, does not always exist, that is the smoothness of $f$ and $g$ does not always imply the smoothness of $u$. Seeing Examples 1.1 and 1.2, it might seem to be impossible to solve the problem. However, we can solve the problem for a certain class of $f$ and $g$ (Theorems 1.3, 1.4 and 1.5).

Throughout this paper, $\kappa$ is a fixed sufficiently small positive number and $s_{*}$ is an integer satisfying

$$
s_{*} \geqslant 14+4 \kappa
$$

((1.2) is necessary for the convergence of our iteration scheme (see Section 2$)$ ). $f \in$ $C^{\prime * *}(\bar{\Omega})$ is a nonnegative function and $g \in C^{s *+2 d}(\bar{\Omega}), 0<d<1$.

We set $S=\{x \in \bar{\Omega}: f(x)=0\}$. We use two kinds of norms $|\cdot|_{k}=\|\cdot\|_{C^{k}(\bar{\Omega})}$ and $\|\cdot\|_{k}=\|\cdot\|_{W^{k}(\Omega)}$. Unless otherwise specified, we use the same notation as in $[0]$. We shall prove the following 
THEOREM 1.3. Assume that $S$ is an isolated point in $\Omega$ and $g_{\nu \nu}>0$ on $S$ for some vector $\nu$. If $\left|\operatorname{det}\left(g_{i j}\right)-f\right|_{s .}$ is sufficiently small, then there is a unique solution $u \in C^{\left[\frac{\operatorname{si}}{2}-2-2 \kappa\right]}(\bar{\Omega})$ of the Dirichlet problem (1.1).

Theorem 1.4. Assume that $S$ is a compact line segment in $\Omega$ and $g_{r r}>0$ for a tangent vector $\tau$ on $S$. If $\left|\operatorname{det}\left(g_{i j}\right)-f\right|_{s .}$ is sufficiently small, then there exists a unique solution $u \in C^{\left[\frac{34}{2}-2-2 \kappa\right]}(\bar{\Omega})$ of the Dirichlet problem (1.1).

Theorem 1.5. Assume that $S$ is a compact $C^{\infty}$ submanifold of $\Omega$ such that $\Omega \backslash S$ is connected and assume that there is a vector $\nu$ satisfying $g_{\nu \nu}>0$ on $S$. If $\left|\operatorname{det}\left(g_{i j}\right)-f\right|_{\text {s. }}$ and $\left|D^{4} g\right|_{0}$ are sufficiently small, then there exists a unique solution $u \in C^{\left[\frac{{ }^{*}}{2}-2-2 \kappa\right]} \bar{\Omega}$ of the Dirichlet problem (1.1).

In each theorem the assumption concerned with $S$ leads us to a priori estimates and the assumption on $f$ and $g$ ensures the existence of approximating solutions (see Section 2). The strange number $\frac{s_{*}}{2}-2-2 \kappa$ comes from our technicalities; the author is wondering whether or not $C^{\left[\frac{\theta_{i}}{2}-2-2 \kappa\right]}$ is a suitable class.

In Section 2 we prove a theorem of Nash-Moser type on the assumption that there exists a sequence of good approximating solutions and a certain type of a priori estimates for linearised operators. Section 3 is devoted to the study of a priori estimates for linear degenerate elliptic operators. We prove Theorem 1.3 - 1.5 in Section 4.

\section{AN iteration Scheme of Nash-MOSER TyPe}

In the first place we shall give several fundamental inequalities which play important roles in the proof of convergence of our iteration shceme. The inequalities

$$
\begin{gathered}
|u|_{i} \leqslant C(i)\|u\|_{i+1+\kappa}, \\
\|u\|_{j} \leqslant C(i, j, k)\|u\|_{i}^{\frac{k-j}{k-i}}\|u\|_{k}^{\frac{j-k}{k-i}} \quad(i<j<k),
\end{gathered}
$$

are well-known. (2.1) and (2.2) are Sobolev and interpolation inequalities respectively. We define operators $S_{\Theta}: W^{i}(\Omega) \rightarrow W^{j}(\Omega)(\Theta \geqslant 1)$ by

$$
S_{\Theta} u(x)=\Theta^{2} \int_{\Omega} \rho(\Theta(x-y)) u(y) d y
$$

where $\rho \in C_{0}^{\infty}\left(\mathbf{R}^{2}\right)$ is a nonnegative function satisfying $\int \rho(x) d x=1 . S_{\Theta}$ are called smoothing operators. It is easy to show that

$$
\begin{gathered}
\left\|\left(I-S_{\Theta}\right) u\right\|_{i} \leqslant C^{\prime}(i, j) \Theta^{i-j}\|u\|_{j} \quad(i<j), \\
\left\|S_{\odot} u\right\|_{i} \leqslant C^{\prime}(i, j) \Theta^{i-j}\|u\|_{j} .
\end{gathered}
$$


Here, in particular, $C(i, i)=1$.

Let $F[u]: W^{k+2}(\Theta) \rightarrow W^{k}(\Omega)\left(k \leqslant s_{*}\right)$ be a map defined by

$$
F[u]=\operatorname{det}\left(u_{i j}\right)-f
$$

We define the linearised operator $L[u]$ and the modified linearised operator $L_{\varepsilon}[u]$ by

$$
L[u] v=a^{i j}[u] v_{i j}=\left.\partial_{t} L[u+t v]\right|_{t=0}
$$

and

$$
L_{\varepsilon}[u] v=a_{\varepsilon}^{i j}[u] v_{i j}=L[u] v+\varepsilon M v
$$

respectively, where $M=\alpha^{i j} \partial_{i} \partial_{j}$ is a second order linear elliptic operator with real constant coefficients.

We shall prove fundamental properties of $F[u], L[u]$ and $L_{e}[u]$.

LEMma 2.1. There is a constant $K \geqslant 0$ such that

$$
\begin{gathered}
|F[u]-F[v]|_{0} \leqslant K\left(|u|_{2}+|v|_{2}\right)|u-v|_{2}, \\
\left\|\partial_{t} L[u+t v] W\right\|_{s} \leqslant K\left[\sum_{\substack{i+j=s \\
i \leqslant s / 2}}\|v\|_{i+3+\kappa}\|w\|_{j+2}+\sum_{\substack{i+j=s \\
j<s / 2}}\|v\|_{i+2}\|w\|_{j+3+\kappa}\right]
\end{gathered}
$$

for $0 \leqslant s \leqslant s_{*}-2$ and $0 \leqslant t \leqslant 1$, and

$$
\|M u\|_{0} \leqslant K\|u\|_{s+2},
$$

for $0 \leqslant s \leqslant s_{*}$.

Proof: (2.5) and (2.7) are clear. (2.6) follows from (2.1) and $\partial_{t} L[u+t v] w=$ $v^{i j} w_{i j}$, where each $v^{k l}$ is the $(k, l)$ - cofactor of the matrix $\left(v_{i j}\right)$.

LEMMA 2.2. $L_{\varepsilon}[u]$ is a formally self-adjoint operator.

Proof: Since

$$
L_{\varepsilon}[u] v=\left(u_{22} v_{1}\right)_{1}-\left(u_{21} v_{2}\right)_{1}-\left(u_{12} v_{1}\right)_{2}+\left(u_{11} v_{2}\right)_{2}+\varepsilon \alpha^{i j} v_{i j}
$$

and $\alpha^{i j}$ are constants, $L_{\epsilon}[u]$ is formally self-adjoint.

LemMA 2.3. If $M u \geqslant 1$, then for $\varepsilon=|F[u]|_{0}$

$$
\operatorname{det}\left(a_{\varepsilon}^{i j}[u]\right) \geqslant f+\varepsilon^{2} \operatorname{det}\left(\alpha^{i j}\right)
$$


Proof: Direct computation gives

$$
\operatorname{det}\left(a_{\varepsilon}^{i j}[u]\right) \geqslant f+\varepsilon(M u-1)+\varepsilon^{2} \operatorname{det}\left(\alpha^{i j}\right)
$$

this proves the lemma.

It is to be noted that the computation in the proofs of Lemmas 2.2 and 2.3 does not remain valid in higher dimensional cases. This is why we have restricted ourselves to two dimensions.

Now we shall construct a sequence which converges to a solution of the equation $F[u]=0$. The convergence requires several assumptions (Assumptions 2.4 -2.6 ).

\section{Assumption 2.4 (the existence of approximating solutions).}

There is a constant $K \geqslant 0$ such that for any $\varepsilon \in(0,1]$ there exist numbers $\delta=\delta(\varepsilon) \in(0,1], \theta=\theta(\varepsilon)>1$ and a function $u_{\varepsilon} \in C^{s_{*}+2, d}(\bar{\Omega})$ satisfying

$$
\begin{gathered}
\left|u_{\varepsilon}\right|_{2} \leqslant K,\left|u_{\varepsilon}\right|_{s} \leqslant K \delta^{-s *} \quad\left(0 \leqslant s \leqslant s_{*}+2\right), \\
\left|F\left[u_{\varepsilon}\right]\right|_{0} \leqslant \varepsilon \theta^{-s *},\left\|F\left[u_{\varepsilon}\right]\right\|_{s} \leqslant \varepsilon \theta^{-s *} \quad\left(0 \leqslant s \leqslant s_{*}\right),
\end{gathered}
$$

and

$$
\left.u_{\varepsilon}\right|_{\partial \Omega}=g
$$

We construct a sequence $\left\{u_{n}\right\}$ as follows:

$$
u_{0}=0, u_{n+1}=u_{n}+v_{n}
$$

where $v_{n} \in C^{s_{*}+2, d}(\bar{\Omega})$ is a solution of the Dirichlet problem

$$
\begin{gathered}
L_{e_{n}}\left[u_{\varepsilon}+\bar{u}_{n}\right] v_{n}=f_{n} \text { in } \Omega, v_{n}=0 \text { on } \partial \Omega \\
\varepsilon_{n}=\left|F\left[u_{\varepsilon}+\tilde{u}_{n}\right]\right|_{0} \\
\tilde{u}_{n}=S_{n} u_{n} \\
f_{0}=-S_{0} F\left[u_{\varepsilon}\right], f_{n}=S_{n-1} R_{n-1}-S_{n} R_{n}+S_{n-1} F\left[u_{\varepsilon}\right]-S_{n} F\left[u_{\varepsilon}\right], \\
R_{0}=0, R_{n}=\sum_{j=0}^{n-1} r_{j}, \\
r_{j}=\left(L_{\varepsilon_{j}}\left[u_{\varepsilon}+u_{j}\right]-L_{\varepsilon_{j}}\left[u_{\varepsilon}+\bar{u}_{j}\right]\right) v_{j}-\varepsilon_{j} M v_{j}+Q_{j} \quad(0 \leqslant j \leqslant n-1), \\
Q_{j}=F\left[u_{e}+u_{j+1}\right]-F\left[u_{\varepsilon}+u_{j}\right]-L\left[u_{\varepsilon}+u_{j}\right] v_{j} \quad(0 \leqslant j \leqslant n-1) .
\end{gathered}
$$

Here $S_{n}=S_{\theta_{n}}$ and $\theta_{n}=(\theta(\varepsilon))^{n}$.

In order to solve the Dirichlet problem (2.13) in $C^{\infty}(\bar{\Omega})$, we need the following 
Assumption 2.5 (the ellipticity of modified linearised operators).

$$
M\left(u_{\varepsilon}+\tilde{u}_{n}\right) \geqslant 1 .
$$

By Lemma 2.3, (2.20) implies $\operatorname{det}\left(a_{\varepsilon_{n}}^{i j}\left[u_{\varepsilon}+\tilde{u}_{n}\right]\right) \geqslant f+\varepsilon_{n}^{2} \operatorname{det}\left(\alpha^{i j}\right)>0$.

The next assumption ensures the convergence of the sequence $\left\{u_{n}\right\}$.

Assumption 2.6(a priori estimates for modified linearised operators).

$$
\left\|v_{n}\right\|_{s} \geqslant K\left\{\left\|f_{n}\right\|_{s}+\sum_{\substack{i+j=s \\ j<s}}\left(1+\left|v_{n}\left\|_{0} \leqslant K\right\| f_{n} \|_{0}, \quad \tilde{u}_{n}\right|_{i+2}\right)\left\|v_{n}\right\|_{j}\right\} \quad\left(0<s \leqslant s_{*}\right),
$$

where $K^{-}=$constant $\geqslant 0$ is independent of $\varepsilon$ and $n$.

In the remainder of this section we estimate $v_{n}, u_{n}, u_{n}-\bar{u}_{n}, r_{n}, f_{n}$ and $\varepsilon_{n}$, and prove that $u_{n}$ converges to a certain function $u_{\infty}$ and that $u_{\varepsilon}+u_{\infty}$ is the solution of $\operatorname{det}\left(u_{i j}\right)=f$ satisfying $\left.u\right|_{\theta \Omega}=g$.

Proposition 2.7. Under Assumptions $2.4-2.6$, if

$$
\begin{gathered}
0<\varepsilon \leqslant\left\{s_{*} K^{-2}+\left(s_{*}+2\right) K\right\}^{-2}, \\
\theta \geqslant 1 / \sqrt{\varepsilon} \delta^{3}, \\
s_{*} \geqslant 4+2 \kappa, \\
5+\kappa \leqslant \sigma \leqslant s_{*},
\end{gathered}
$$

then

$$
\begin{gathered}
\left\|v_{0}\right\|_{s} \leqslant \frac{\sqrt{\varepsilon}}{2} \theta^{s-\sigma} \quad\left(0 \leqslant s \leqslant s_{*}\right), \\
\left\|r_{0}\right\|_{s} \leqslant C_{1} \varepsilon \theta^{s-\sigma} \quad\left(0 \leqslant s \leqslant s_{*}-s\right),
\end{gathered}
$$

where $C_{1}=$ constant $\geqslant 0$ depends only on $s_{*}$ and $K^{-}$.

Proposition 2.8. Under Assumptions $2.4-2.6$, if

$$
0<\varepsilon \leqslant \min \left[\left\{s_{*} K^{2}+\left(s_{*}+2\right) K\right\}^{-2},\left(2 K C_{2}\right)^{-2}, \alpha, \beta,\right]
$$

where

$$
\alpha=\max _{0 \leqslant s \leqslant s .}\left\{K\left\{2 C_{2}+\sum_{\substack{i+j=s \\ j<s}}(1+K+C(i+2) C(i+3+\kappa,, \sigma+\kappa))\right\}\right\}^{-2},
$$


and

$$
\beta=\left\{K^{\prime}(0)\left(2 C^{\prime}\left(1+\kappa, s_{*}-2\right)+1\right)\right\}^{-2}
$$

$$
\begin{aligned}
\theta \geqslant \max & \left(1 / \sqrt{\varepsilon} \delta^{3}, 2^{1 / \kappa}\right), \\
s_{*} & \geqslant 14+4 \kappa \\
\sigma & =\frac{s_{*}}{2}-1, \\
\tau & =\sigma-2,
\end{aligned}
$$

then we have

$$
\begin{gathered}
\left\|v_{j-1}\right\|_{s} \leqslant \frac{\sqrt{\varepsilon}}{2} \theta_{j-1}^{s-\sigma} \quad\left(0 \leqslant s \leqslant s_{*}\right), \\
\left\|u_{j}\right\|_{s} \leqslant \begin{cases}\sqrt{\varepsilon} \quad \text { if } s \leqslant \sigma-k, \\
\sqrt{\varepsilon} \theta_{j}^{s-\sigma} \quad \text { if } s \geqslant \sigma-k,\end{cases} \\
\left\|u_{j}-\bar{u}_{j}\right\|_{s} \leqslant C_{0} \sqrt{\varepsilon} \theta_{j}^{s-\sigma} \quad\left(0 \leqslant s \leqslant s_{*}\right), \\
\left\|r_{j-1}\right\|_{s} \leqslant C_{1} \varepsilon \theta_{j-1}^{s-\sigma} \quad\left(0 \leqslant s \leqslant s_{*}-2\right), \\
\left\|f_{j}\right\|_{s} \leqslant C_{2} \varepsilon \theta_{j}^{s-\sigma} \quad\left(0 \leqslant s \leqslant s_{*}\right), \\
\varepsilon_{j} \leqslant C_{3} \sqrt{\varepsilon} \theta_{j}^{r-\sigma},
\end{gathered}
$$

where $v_{-1}=0, r_{-1}=0$ and constants $C_{i} \geqslant 0$ depend only on $\kappa, s_{*}, \sigma$ and $K^{-}$.

REMARK: By virtue of the interpolation inequality $(2.2),(2.27)-(2.28)$ and $(2.34)_{j}$ - $(2.39)_{j}$ remain valid for real $s$, if we modify the constants $C_{0}-C_{3}$ appropriately.

Before proving Propositions 2.7 and 2.8, we shall prove the main theorem of this section.

Theonem 2.9. Under the same assumptions as Proposition 2.8, there exists a function $u_{\infty} \in W^{\frac{e^{*}-1-\kappa}{2}}(\Omega)$ such that

$$
\operatorname{det}\left(\left(u_{\varepsilon}+u_{\infty}\right)_{i j}\right)=f \quad \text { in } \quad \Omega, u_{\varepsilon}+u_{\infty}=g \quad \text { on } \quad \partial \Omega
$$

and

$$
\left\|u_{\infty}\right\|_{\frac{\ddot{*}}{2}-1-\kappa} \leqslant \sqrt{\varepsilon}
$$

Proof: By Proposition 2.8,

$$
\left\|u_{i}-u_{j}\right\|_{\frac{\mu^{*}-1-\kappa}{2}} \leqslant \sum_{\nu=j}^{i-1}\left\|v_{\nu}\right\|_{\frac{\alpha_{*}-1-\kappa}{2}} \leqslant \frac{\sqrt{\varepsilon}}{2} \sum_{\nu=j}^{i-1}\left(\theta^{-\kappa}\right)^{\nu} \rightarrow 0
$$


as $i, j \rightarrow \infty, i>j$. Hence, there is a function $u_{\infty} \in W^{\frac{s_{*}^{*}}{2}-1-\kappa}(\Omega)$ satisfying $u_{n} \rightarrow u_{\infty}$ in $W^{\frac{\theta_{*}^{*}}{2}-1-\kappa}(\Omega)$. Since $W^{\frac{s_{*}^{*}}{2}-1-\kappa}(\Omega) \hookrightarrow C^{2}(\bar{\Omega}), u_{\infty} \in C^{2}(\bar{\Omega})$ and $u_{n} \rightarrow u_{\infty}$ in $C^{2}(\bar{\Omega})$.

On the other hand, direct computation gives, by (2.13) - (2.19),

$$
F\left[u_{\varepsilon}+u_{n+1}\right]=\left(1-S_{n}\right) F\left[u_{\varepsilon}\right]+\left(1-S_{n}\right) R_{n}+r_{n}
$$

Combining (2.42) with $(2.3)$ and $(2.37)_{j}$, we can show that $F\left[u_{\varepsilon}+u_{n}\right] \rightarrow 0$ in $W^{\frac{\ell_{*}^{*}}{2}-1-\kappa}(\Omega)$; this implies $F\left[u_{\varepsilon}+u_{n}\right] \rightarrow 0$ in $C^{0}(\bar{\Omega})$, since $W^{\frac{\varepsilon_{*}^{*}}{2}-1-K^{-}}(\Omega) \rightarrow C^{0}(\bar{\Omega})$.

Therefore, $u_{\infty} \in W^{\frac{\sigma_{*}^{*}}{2}-1-\kappa}(\Omega)$ and $F\left[u_{\varepsilon}+u_{\infty}\right]=0$. Since $\left.u_{\varepsilon}\right|_{\theta \Omega}=g$ and $\left.u_{n}\right|_{\partial \Omega}=\left.\sum_{j=0}^{n-1} v_{j}\right|_{\partial \Omega}=0$, we have $\left.\left(u_{\epsilon}+u_{\infty}\right)\right|_{\partial \Omega}=g$. By $(2.35)_{j},\left\|u_{\infty}\right\|_{\frac{\mu_{-}-1-\kappa}{2}}=$ $\lim _{n \rightarrow \infty}\left\|u_{n}\right\|_{\frac{s_{*}-1-\kappa}{2}} \leqslant \sqrt{\varepsilon}$.

Proof of Proposition 2.7: (2.21), (2.16), (2.4), (2.10) and $s_{*} \geqslant \sigma$ give

$$
\left\|v_{0}\right\|_{0} \leqslant \frac{\sqrt{\varepsilon}}{2} \theta^{-\sigma}
$$

We suppose that

$$
\left\|v_{0}\right\|_{j} \leqslant \frac{\sqrt{\varepsilon}}{2} \theta^{j-\sigma} \quad(0 \leqslant j<s)
$$

and estimate $\left\|v_{0}\right\|_{\text {. }}$. By (2.22), we have

$$
\left\|v_{0}\right\|_{s} \leqslant K^{-}\left\{\left\|f_{0}\right\|_{s}+\sum_{\substack{i+j=s \\ j<s}}\left(1+\left|u_{\varepsilon}\right|_{i+2}\right)\left\|v_{0}\right\|_{j}\right\} .
$$

(2.16), (2.4) and $s_{*}+s \geqslant \sigma$ give

$$
\left\|f_{0}\right\|_{s} \leqslant \varepsilon \theta^{s-\sigma}
$$

(2.24) implies

$$
1 \leqslant \sqrt{\varepsilon} \theta^{i} \quad(0<i \leqslant s) .
$$

By (2.9) and (2.24), we have

$$
\left|u_{e}\right|_{i_{+}} \leqslant K^{-} \sqrt{\varepsilon} \theta^{i} \quad(0<i \leqslant s) .
$$


Combining (2.43) - (2.47), we obtain

$$
\left\|v_{0}\right\|_{s} \sqrt{\varepsilon}\left\{s_{*} K^{2}+\left(s_{*}+2\right) K\right\} \frac{\sqrt{\varepsilon}}{2} \theta^{s-\sigma} ;
$$

this implies, by $(2.23)$,

$$
\left\|v_{0}\right\|_{s} \leqslant \frac{\sqrt{\varepsilon}}{2} \theta^{s-\sigma} .
$$

Thus (2.27) is proved by induction.

Next we prove (2.28). (2.18) gives

$$
r_{0}=-\varepsilon_{0} M v_{0}+Q_{0} .
$$

$\left(2.7,(2.14),(2.10)\right.$ and $s_{*} \geqslant 2$ show that

$$
\left\|\varepsilon_{0} M v_{0}\right\|_{s} \leqslant \frac{1}{2} K \varepsilon \theta^{s-\sigma} .
$$

Since

$$
Q_{0}=\int_{0}^{1}\left\{\int_{0}^{t} \partial_{s} L\left[u_{\varepsilon}+s v_{0}\right] v_{0} d s\right\} d t,
$$

we have, by $(2.6), s_{*} \geqslant 4+2 \kappa$ and $\sigma \geqslant 5+\kappa$,

$$
\left\|Q_{0}\right\|_{\theta} \leqslant \frac{1}{4}\left(s_{*}+1\right) K \varepsilon \theta^{s-\sigma} .
$$

Combining (2.48) - (2.50), we obtain (2.28).

Proof of Proposition 2.8: $(2.34)_{0}-(2.39)_{0}$. $(2.34)_{0}-(2.37)_{0}$ are clear. (2.16), (2.4), (2.10) and $s_{*}+s \geqslant \sigma$ show $(2.38)_{0} \cdot(2.39)_{0}$ follows from $(2.14),(2.10)$ and $s_{*}+s \geqslant \sigma$.

$(2.34)_{j \leqslant n}-(2.39)_{j \leqslant n} \Rightarrow(2.34)_{n+1} \cdot(2.21),(2.38)_{n}$ and (2.29) give

$$
\left\|v_{n}\right\|_{0} \leqslant \frac{\sqrt{\varepsilon}}{2} \theta_{n}^{-\sigma} \text {. }
$$

We suppose that

$$
\left\|v_{n}\right\|_{j} \leqslant \frac{\sqrt{\varepsilon}}{2} \theta_{n}^{j-\sigma} \quad(0 \leqslant j<s)
$$

and estimate $\left\|v_{n}\right\|_{0}$. By $(2.22)$, we have

$$
\left\|r_{n}\right\| \leqslant K\left\{\left\|r_{n}\right\|_{o}+\sum_{\substack{i+j=s \\ j<s}}\left(1+\left|u_{\varepsilon}+\bar{u}_{n}\right|_{i+2}\right)\left\|v_{n}\right\|_{j}\right\} .
$$


Clombining $(2.52)$ with $(2.38)_{n},(2.46),(2.47),(2.1),(2.4),(2.35)_{n}$ and $(2.51)$, we obtain

$$
\left\|v_{n}\right\|_{s} \leqslant \sqrt{\varepsilon} \Pi\left\{2 C_{2}+\sum_{\substack{i+j=s \\ j<s}}(1+K+C(i+2) C(i+3+\kappa, \sigma+\kappa))\right\} \frac{\sqrt{\varepsilon}}{2} \theta_{n}^{s-\sigma}
$$

this implies by $(2.29)$,

$$
\left\|v_{n}\right\|_{s} \leqslant \frac{\sqrt{\varepsilon}}{2} \theta_{n}^{s-\sigma}
$$

Thus $(2.34)_{n+1}$ is proved.

$(2.34)_{j \leqslant a}-(2.39)_{j \leqslant n} \Rightarrow(2.35)_{n+1}$. Since $u_{n+1}=\sum_{j=0}^{n} v_{j}$, we have, by $(2.34)_{n+1}$,

$$
\left\|u_{n+1}\right\|_{s} \leqslant \frac{\sqrt{\varepsilon}}{2} \sum_{j=0}^{n} \theta_{j}^{s-\sigma}
$$

Direct computation gives, by (2.30),

$$
\frac{\sqrt{\varepsilon}}{2} \sum_{j=0}^{n} \theta_{j}^{s-\sigma} \leqslant \begin{cases}\sqrt{\varepsilon} & (s \leqslant \sigma-\kappa) \\ \sqrt{\varepsilon} \theta_{n+1}^{s-\sigma} & (s \geqslant \sigma+\kappa) .\end{cases}
$$

$(2.34)_{j \leqslant n}-(2.39)_{j \leqslant n} \Rightarrow(2.36)_{n+1}$ It follows immediately from $(2.3),(2.4)$ and $(2.34)_{n+1}$ that

$$
\left\|u_{n+1}-\tilde{u}_{n+1}\right\|_{s} \leqslant \begin{cases}C(s, \sigma+\kappa) \sqrt{\varepsilon} \theta_{n+1}^{s-\sigma} & (s<\sigma+\kappa), \\ 2 \sqrt{\varepsilon} \theta_{n+1}^{s-\sigma} & (s \geqslant \sigma+\kappa) .\end{cases}
$$

So we have only to take

$$
C_{0}^{\prime}=\max \left[\max _{0 \leqslant s<\sigma+\kappa} C(s, \sigma+\kappa), 2\right] .
$$

$(2.34)_{j<u}-(2.39)_{j \leqslant n} \Rightarrow(2.37)_{n+1}$. Since

$$
\left(L_{\varepsilon_{n}}\left[u_{\varepsilon}+u_{n}\right]-L_{\varepsilon_{n}}\left[u_{\varepsilon}+\tilde{u}_{n}\right]\right) v_{n}=\int_{0}^{1} \partial_{t} L_{\varepsilon_{n}}\left[u_{\varepsilon}+\tilde{u}_{n}+t\left(u_{n}-\tilde{u}_{n}\right)\right] v_{n} d t,
$$

we have, by $(2.6), s_{*} \geqslant 4+2 \kappa$ and $\sigma \geqslant 5+\kappa$,

$$
\left\|\left(L_{e_{n}}\left[u_{\varepsilon}+u_{n}\right]-L_{e_{n}}\left[u_{\varepsilon}+\bar{u}_{n}\right]\right) v_{n}\right\|_{9} \leqslant \frac{1}{2}\left(s_{*}+1\right) K C_{0} \varepsilon \theta_{n}^{s-\sigma}
$$


$(2.7),(2.39)_{n}$ and $(2.33)$ give

$$
\left\|\varepsilon_{n} M v_{n}\right\|_{s} \leqslant \frac{1}{2} K C_{3} \varepsilon \theta_{n}^{s-\sigma}
$$

Since

$$
Q_{n}=\int_{0}^{1}\left\{\int_{0}^{t} \partial_{s} L\left[u_{e}+u_{n}+s v_{j}\right] v_{j} d s\right\} d t,
$$

we obtain, by $\left(2.6, s_{*} \geqslant 4+2 \kappa\right.$ and $\sigma \geqslant 5+\kappa$,

$$
\left\|Q_{n}\right\|_{s} \leqslant \frac{1}{4}\left(s_{*}+1\right) K \varepsilon \theta_{n}^{s-\sigma} .
$$

By (2.18), (2.53) - (2.55) imply

$$
\left\|r_{n}\right\|_{s} \leqslant C_{1}^{\prime} \varepsilon \theta_{n}^{s-\sigma}
$$

where

$$
C_{1}=\frac{1}{2}\left(s_{*}+1\right) K C_{0}+\frac{1}{2} K C_{3}+\frac{1}{4}\left(s_{*}+1\right) K .
$$

$(2.34)_{j \leqslant n}-(2.39)_{j \leqslant n} \Rightarrow(2.38)_{n+1}$. We note that, by $(2.16)$,

$$
f_{n+1}=S_{n} R_{n}-S_{n+1} R_{n+1}+S_{n} F\left[u_{\varepsilon}\right]-S_{n+1} F\left[u_{\varepsilon}\right] .
$$

(2.4), $(2.37)_{j \leqslant n},(2.32)$ and $\theta \geqslant 2^{1 / \sigma}$ give

$$
\begin{gathered}
\left\|S_{n} R_{n}\right\|_{s} \leqslant 2 C_{1} C\left(s, s_{*}-2\right) \varepsilon \theta_{n+1}^{s-\sigma}, \\
\left\|S_{n+1} R_{n+1}\right\|_{s} \leqslant C_{1} C\left(s, s_{*}-2\right) \varepsilon \theta_{n+1}^{s-\sigma} .
\end{gathered}
$$

(2.4), (2.10) and $s_{*}+s \geqslant \sigma$ show that

$$
\begin{gathered}
\left\|S_{n} F\left[u_{e}\right]\right\|_{s} \leqslant C(s, \sigma) \varepsilon \theta_{n+1}^{s-\sigma}, \\
\left\|S_{n+1} F\left[u_{e}\right]\right\|_{s} \leqslant C(s, \sigma) \varepsilon \theta_{n+1}^{s-\sigma} .
\end{gathered}
$$

Combining (2.56) - (2.60), we obtain

$$
\left\|f_{n+1}\right\|_{0} \leqslant C_{2} \varepsilon \theta_{n+1}^{s-\sigma} \text {. }
$$

where

$$
C_{2}=3 C_{1} \max _{0 \leqslant s \leqslant s_{*}} C\left(s, s_{*}-2\right)+2 \max _{0 \leqslant s \leqslant s_{*}} C(s, \sigma) .
$$


$(2.34)_{j \leqslant n}-(2.39)_{j \leqslant n} \Rightarrow(2.39)_{n+1} \cdot(2.14)$ and $(2.42)$ give

$$
\begin{gathered}
\varepsilon_{n+1} \leqslant\left|\left(I-S_{n}\right) F\left[u_{\varepsilon}\right]\right|_{0}+\left|\left(I-S_{n}\right) R_{n}\right|_{0} \\
+\left|r_{n}\right|_{0}+\left|F\left[u_{\varepsilon}+\tilde{u}_{n}\right]-F\left[u_{\varepsilon}+u_{n}\right]\right|_{0} .
\end{gathered}
$$

By $(2.1),(2.3),(2.10), s_{*} \geqslant \sigma$ and $\tau \geqslant 1+\kappa$, we have

$$
\left|\left(I-S_{n}\right) F\left[u_{\varepsilon}\right]\right|_{0} \leqslant C^{\prime}(0) C^{\prime}\left(1+\kappa, s_{*}\right) \varepsilon \theta_{n+1}^{\tau-\sigma}
$$

By $(2.1),(2.3),(2.37) j \leqslant n,(2.32), \theta \geqslant 2^{1 / \sigma}$ and $\tau \geqslant 1+\kappa$, we obtain

$$
\left|\left(I-S_{n}\right) R_{n}\right|_{0} \leqslant 2 C_{1}^{\prime} C(0) C\left(1+\kappa, s_{*}-2\right) \varepsilon \theta_{n+1}^{\tau-\sigma} .
$$

(2.1), Proposition 2.7 and $\tau \geqslant 1+\kappa$ give

$$
\left|r_{0}\right|_{0} \leqslant C_{1} C(0) \varepsilon \theta^{r-\sigma}
$$

$(2.1),(2.37)_{n+1}$ and $\tau \geqslant \frac{\sigma}{2}+1+\kappa$ show that

$$
\left|r_{n}\right|_{0} \leqslant C_{1} C^{\prime}(0) \varepsilon \theta_{n+1}^{\tau-\sigma} \quad(n \geqslant 1)
$$

From $(2.5),(2.1),(2.9),(2.35)_{n+1}, 3+\kappa \leqslant \sigma-\kappa,(2.36)_{n+1}$ and $\tau \geqslant 3+\kappa$

$$
\left|F\left[u_{\varepsilon}+\tilde{u}_{n+1}\right]-F\left[u_{\epsilon}+u_{n+1}\right]\right|_{0} \leqslant 2 K C(2)(K+C(2) \sqrt{\varepsilon}) C_{0} \sqrt{\varepsilon} \theta_{n+1}^{r-\sigma}
$$

follows. Combining (2.61) - (2.66), we obtain

$$
\varepsilon_{n+1} \leqslant C_{3} \sqrt{\varepsilon} \theta_{n+1}^{\tau-\sigma}
$$

where

$$
C_{3}=C(0) C\left(1+K, s_{*}\right)+\frac{1}{K} C_{1}+2 K C_{0}^{\prime} C(2)(K+C(2))
$$

Here we used (2.29), in other words, $\sqrt{\varepsilon} \leqslant\left\{K C(0)\left(2 C\left(1+K, s_{*}-2\right)+1\right)\right\}^{-1}$.

In order to determine the constants $C_{1}, C_{2}$ and $C_{3}$ explicitly, we have only to regard the three equations which define $C_{1}, C_{2}$ and $C_{3}$ as simultaneous algebraic equations with respect to $C_{1}, C_{2}$ and $C_{3}$ and solve them.

\section{A Priori estimates for linear operators}

Let $L$ be a degenerate elliptic operator of the form

$$
L=a^{i j} \partial_{i} \partial_{j}
$$


with real coefficients $a^{i j}=a^{j i} \in C^{\infty}(\bar{\Omega})$. Here "degenerate elliptic" means $a^{i j}(x) \xi_{i} \xi_{j}$ $\geqslant 0$ for any $(x, \xi) \in \bar{\Omega} \times \mathbf{R}^{2}$. For simplicity we use the following notation: $\lambda(x)$ is a continuous function defined in $\Omega$ satisfying

$$
\inf _{|\xi|=1} a^{i j}(x) \xi_{i} \xi_{j} \geqslant \lambda(x) \geqslant 0,
$$

$S$ denotes the set of all zero points of $\lambda(x)$ in $\Omega$,

$$
\Lambda_{k}=\max _{i, j}\left|D^{k} a^{i j}\right|_{0}
$$

and

$$
A(k)=\max \left\{\max _{i, j}\left|a^{i j}\right|_{k}, \inf _{x} \lambda(x), 1\right\} .
$$

Unless otherwise specified, $C, C^{\prime}, C_{1}$ and $C_{1}^{\prime}$ denote nonnegative constants independent of $L$.

First we shall give four fundamental lemmas. For $t \geqslant 1$ and $\Phi \in C^{\infty}(\bar{\Omega})$ satisfying $\{x \in \Omega: \Phi(x)=0\} \neq \emptyset$ and $D \Phi \neq 0$, we put

$$
U(\Phi, t)=\{x \in \Omega:|\Phi(x)|<1 / t\} .
$$

Lemma 3.1. Assume that $L$ is formally self-adjoint. Then there is a constant $C$ independent of $L, \Phi$ and $t$ such that

$$
\begin{array}{r}
t^{2} \int\left(a^{i j} \Phi_{i} \Phi_{j}\right) u^{2} d x \leqslant C\left[\int|L u \cdot u| d x+t \int\left|a^{i j} \Phi_{i j}\right| u^{2} d x\right] \\
\left(u \in C^{\infty}(\overline{U(\Phi, t)}),\left.u\right|_{\partial U(\Phi, t)}=0\right) .
\end{array}
$$

Proof: For $u \in C^{\infty}(U(\Phi, t))$ satisfying $\left.u\right|_{\partial U(\Phi, t 6)}=0$, we put

$$
v=\left(T-e^{t \Phi}\right)^{-1} u, \quad T=\text { constant }>2 e .
$$

Direct computation gives

$$
L u=\left(T-e^{t \Phi}\right) a^{i j} v_{i j}-e^{t \Phi}\left\{2 t a^{i j} \Phi_{i} v_{j}+\left(t^{2} a^{i j} \Phi_{i} \Phi_{j}+t a^{i j} \Phi_{i j}\right) v\right\} .
$$

Hence, integrating $\left(T-e^{t \Phi}\right)^{-1} L u \cdot v$ by parts, we obtain

$$
\begin{gathered}
\int\left(T-e^{t \Phi}\right)^{-1} L u \cdot v d x \\
=-\int a^{i j} v_{i} v_{j} d x+\frac{1}{2} \int a_{i j}^{i j} v^{2} d x-2 \int a^{i j}\left(t e^{t \Phi}\left(T-e^{t \Phi}\right)^{-1} \Phi_{i} v\right) v_{j} d x \\
-t^{2} \int e^{t \Phi}\left(T-e^{t \Phi}\right)^{-1}\left(a^{i j} \Phi_{i} \Phi_{j}\right) v^{2} d x-t \int e^{t \Phi}\left(T-e^{t \Phi}\right)^{-1}\left(a^{i j} \Phi_{i j}\right) v^{2} d x
\end{gathered}
$$


Since $a_{i j}^{i j}=0$ and

$$
\left|2 a^{i j}\left(t e^{t \Phi}\left(T-e^{t \Phi}\right) \Phi_{i} v\right) \Phi_{j}\right| \leqslant t^{2} e^{2 t \Phi}\left(T-e^{t \Phi}\right)^{-2}\left(a^{i j} \Phi_{i} \Phi_{j}\right) v^{2}+a^{i j} v_{i} v_{j}
$$

we have

$$
\begin{array}{r}
\int\left(T-e^{t \Phi}\right)^{-2} L u \cdot u d x \leqslant-t^{2} \int e^{t \Phi}\left(T-2 e^{t \Phi}\right)\left(T-e^{t \Phi}\right)^{-4}\left(a^{i j} \Phi_{i} \Phi_{j}\right) u^{2} d x \\
-t \int e^{t \Phi}\left(T-e^{t \Phi}\right)^{-3}\left(a^{i j} \Phi_{i j}\right) u^{2} d x
\end{array}
$$

Here we note that $e^{-1} \leqslant \epsilon^{t \Phi} \leqslant \epsilon, T-2 \epsilon \leqslant T-2 \epsilon^{t \Phi} \leqslant T-2 e^{-1}$ and $\left(T-\epsilon^{-1}\right)^{-1} \leqslant$ $\left(T_{c}^{t+}\right)^{-1} \leqslant(T-e)^{-1}$ in $U(\Phi, t)$. Combining $(3.3)$ with these inequalities, we obtain (3.1).

For $\varepsilon>0$ we define a set $S_{\varepsilon}$ by

$$
S_{\varepsilon}=\{x \in \bar{\Omega}: \text { dist }(x, S)>\varepsilon\} \text {. }
$$

Lemma 3.2. Assume that $L$ is formally self-adjoint, $S$ is a compact $C^{\infty}$ submanifold of $\Omega$ and $\Omega \backslash S$ is connected. Then there exists a function $\mu \in L^{\infty}(\Omega)$ such that $\mu=0$ on $S, \inf _{\Omega \backslash S_{\varepsilon}} \mu>0$ for any sufficiently small $\varepsilon>0$ and

$$
\int \mu u^{2} d x+\int \lambda|D u|^{2} d x \leqslant C\|L u\|_{0}\|u\|_{0} \quad\left(u \in C^{\infty}(\bar{\Omega}),\left.u\right|_{\theta \Omega}=0\right) .
$$

Proof: Since $L$ is formally self-adjoint, it will suffice to prove

$$
\int \mu u^{2} d x \leqslant C \int \lambda|D u|^{2} d x \quad\left(u \in C^{\infty}(\bar{\Omega}),\left.u\right|_{\theta \Omega}=0\right) .
$$

First, we fix a point $p \in \overline{\Omega \backslash S}$ arbitrarily. By virtue of the fundamental theorems of ordinary differential equations, we can construct a family of curves $c(t ; x) \in C^{\infty}\left(\left[0, T_{p}\right] \times U_{p}\right)$ such that $c(0 ; x)=x, c(t ; x) \notin S$ for $0<t<T_{p}$ when $x \in \overline{\Omega \backslash S}, c\left(T_{p} ; x\right) \notin \bar{\Omega},|\dot{c}(t ; x)| \equiv 1, C(t ; \cdot)$ is a lucal $C^{\infty}$ diffeomorphism defined in $U_{p}$, for any fixed $t$, and such that $\sup _{x \in U_{p}} \tau_{x}<\infty$, where $U_{p}$ is a sufficiently small open neighbourhood of $p, T_{p}$ is a positive constant and $\tau_{x}=\inf \{t \geqslant 0: c(1 ; x) \notin \bar{\Omega}\}$. We define a function $\mu_{p}(x)$ by

$$
\mu_{p}(x)=\inf \left\{\lambda(c(t ; x)): 0 \leqslant t \leqslant \tau_{x}\right\}
$$

for $x \in U_{p}$. For $u \in C^{\infty}(\bar{\Omega})$ satisfying $\left.u\right|_{\partial \Omega}=0$, since

$$
u(x) u(c(0 ; x))-u\left(c\left(\tau_{x} ; x\right)\right)=-\int_{0}^{\tau_{x}} D u(c(t ; x)) \cdot \dot{c}(t ; x) d t
$$


we have

$$
|u(x)|^{2} \leqslant C \int_{0}^{\tau_{x}}|D u(c(t ; x))|^{2} d t
$$

By multiplying (3.7) by $\mu_{p}(x)$ and using (3.6), we obtain

$$
\mu_{p}(x)|u(x)|^{2} \leqslant C \int_{0}^{T_{x}} \lambda(c(t ; x))|D u(c(t ; x))|^{2} d t
$$

this implies

$$
\int_{U_{p}} \mu_{p} u^{2} d x \leqslant C \int_{\Omega} \lambda|D u|^{2} d x
$$

Secondly, we note that the above argument ensures the existence of a finite number of points $p_{1}, \ldots, p_{N}$ such that $\overline{\Omega \backslash S} \subset \bigcup_{i=1}^{N} U_{p_{i}}$ and

$$
\int_{U_{p_{i}}} \mu_{p_{i}} u^{2} d x \leqslant C \int_{\Omega} \lambda|D u|^{2} d x
$$

Therefore, we have only to define $\mu(x)$ by

$$
\mu(x)= \begin{cases}\min \left\{\mu_{p_{i}}(x): x \in U_{p_{i}}, 1 \leqslant i \leqslant n\right\}, & \text { if } x \in \Omega \backslash S \\ 0, & \text { if } x \in S .\end{cases}
$$

$(3.5)$ is proved.

\section{LEMMA 3.3 .}

$$
\sum_{k}\left\|\left[\partial_{k}, L\right] u\right\|_{0}^{2} \leqslant C^{\prime} A_{2}\left(\|L u\|_{1}\|u\|_{1}+A_{2}\|u\|_{1}^{2}\right) \quad\left(u \in C_{0}^{\infty}(\Omega)\right)
$$

Lemma 3.4. For a fixed $\chi \in C^{\infty}(\Omega)$ satisfying supp $|D \chi| \subset \Omega$

$$
\|[x, L] u\|_{0}^{2} \leqslant C: A_{0}\left(\|L u\|_{0}\|u\|_{0}+A(2)\|u\|_{0}^{2}\right) \quad\left(u \in C^{\infty}(\Omega)\right)
$$

Here $\left[\partial_{k}, L\right] u=\partial_{k} L u-L \partial_{k} u$ and $[\chi, L] u=\chi L u-L \chi u$.

PRoOf of LEMMA 3.3: It is well-known that

$$
\sum_{k}\left(a_{k}^{i j} u_{i j}\right)^{2} \leqslant C A_{2} \sum_{l} a^{i j} u_{l i} u_{l j}
$$


for any $u \in C_{0}^{\infty}(\Omega)$ (see [13]; Lemma 1.7.1 and also [2]; Lemma 1.3). This gives

$$
\sum_{k}\left\|\left[\partial_{k}, L\right] u\right\|_{0}^{2} \leqslant C \cdot A_{2} \sum_{l} \int a^{i j} u_{l i} u_{l j} d x .
$$

Integrating by parts carefully, we have

$$
\sum_{k}\left\|\left[\partial_{k}, L\right] u\right\|_{0}^{2} \leqslant C A_{2}\left(\|L u\|_{1}\|u\|_{1}+\sum_{k}\left\|\left[\partial_{k}, L\right] u\right\|_{0}\|u\|_{0}+A_{2}\|u\|_{1}^{2}\right) .
$$

This completes the proof.

ProOF OF Lemma 3.4: Let us consider a cut-off function $\bar{\chi} \in C_{0}^{\infty}(\Omega)$ satisfying $0 \leqslant \tilde{\chi} \leqslant 1$ and $\partial_{i} \chi \subset \subset \bar{\chi}$ for any $i$. We define an operator $\tilde{L}=\tilde{a}^{i j} \partial_{i} \partial_{j}$ by $\bar{L}=$ $\bar{\chi} L$. Since $[\chi, \bar{L}] u=[\chi, L] u$ and $\|\tilde{L} u\|_{0} \leqslant\|L u\|_{0}$, it will suffice to prove a modified inequality which we get by substituting $\tilde{L}$ into $L$ in (3.9). It is easy to prove

$$
\sum_{i}\left(\tilde{a}^{i j} u_{i j}\right)^{2} \leqslant A_{0} \tilde{a}^{k l} u_{k} \cdot u_{l}
$$

(see [13]; Corollary of Lemma 1.7.1 and also [2]; Lemma 1.2); this shows

$$
\|[\chi, \tilde{L}] u\|_{0}^{2} \leqslant C^{\prime} A_{0}\left(\int \tilde{a}^{i j} u_{i} u_{j} d x+A_{0}\|u\|_{0}^{2}\right) .
$$

Therefore, integrating $\int \vec{a}^{i j} u_{i} u_{j} d x$ by parts, we obtain

$$
\|[\chi, \bar{L}] u\|_{0}^{2} \leqslant C A_{0}\left(\|\bar{L} u\|_{0}\|u\|_{0}+A(2)\|u\|_{0}^{2}\right)
$$

(3.9) is proved.

Now we give the main theorem of this section which follows from Lemmas $3.2-3.4$.

THEOREM 3.5. Assume that $L$ is formally self-adjoint, $S$ is a compact $C^{\infty}$ submanifold of $\Omega$ and $\Omega \backslash S$ is connected. Then for any nonnegative integer $s$ there is a constant $C_{s, \lambda}$, which depends on $s$ and $\lambda(x)$ but does not depend on $L$, such that

$$
\begin{gathered}
\|u\|_{0} \leqslant C_{0, \lambda}\|L u\|_{0} \quad\left(u \in C^{\infty}(\bar{\Omega}),\left.u\right|_{\partial \Omega}=0\right), \\
\|u\|_{1} \leqslant C_{1, \lambda}^{\prime}\left(\|L u\|_{1}+\|u\|_{0}\right) \quad\left(u \in C^{\infty}(\bar{\Omega}),\left.u\right|_{\partial \Omega}=0\right)
\end{gathered}
$$

and

$$
\|u\|_{s} \leqslant C_{x, \lambda}\left(\|L u\|_{s}+\sum_{\substack{i+j \leqslant s \\ j<s}}^{*} A(i+2)\|u\|_{j}\right) \quad\left(u \in C^{\prime \infty}(\bar{\Omega}),\left.u\right|_{\theta \Omega}=0, s \geqslant 2\right)
$$


provided that there is an open neighbourhood $U$ of $S$ in $\Omega$ such that

$$
\|u\|_{0} \leqslant K^{-}\|L u\|_{0} \quad\left(u \in C_{0}^{\infty}(U)\right),
$$

$0 \leqslant K A_{2}<<1$ and $A(2) \leqslant 1$.

Here $\sum_{i+j \leqslant t}^{*}$ means $\sum_{\substack{i+j \leqslant t \\ i+2 \leqslant \max (t, 2)}}$, "<<1" means "to be sufficiently small". The fact that the constants $C_{s, \lambda}$ depend on $\lambda(x)$ but do not depend on $L$ is very important (see Assumption 2.5 and Section 4). Theorem 3.5 says that if you want to prove a priori extimates (3.10) - (3.12), you have only to prove a simple $L^{2}$ estimate (3.13) near $S$ where $L$ is degenerating. The key estimates of Assumption 2.6 will follow from Lemma 3.1 and Theorem 3.5 (compare Section 4).

The proof of Theorem 3.5 requires the following four lemmas.

Lemma 3.6.

$$
\sum_{k}\left\|\left[\partial_{k}, L\right] u\right\|_{s}^{2} \leqslant C_{s}\left(A_{2}\|L u\|_{s+1}+\sum_{i+j \leqslant s+1}^{*} A_{i+2}^{2}\|u\|_{j}^{2}\right),\left(u \in C_{0}^{\infty}(\Omega)\right) .
$$

LEMMA 3.7. For a fixed $\chi \in C^{\infty}(\Omega)$ satisfying supp $|D \chi| \subset \Omega$

$$
\|[x, L] u\|_{。} \leqslant C_{s}\left(A(2)\|L u\|_{s}\|u\|_{s}+\sum_{i+j \leqslant s}^{*} A^{2}(i+2)\|u\|_{j}^{2}\right),\left(u \in C^{\infty}(\Omega)\right) .
$$

LEMMA 3.8. Assume that

$$
\|u\|_{0} \leqslant K\|L u\|_{0},\left(u \in C_{0}^{\infty}(\Omega)\right) .
$$

Then for any nonnegative integer $s$ there exists a constant $C$, independent of $L$ and $K$ such that

$$
\|u\|_{s} \leqslant K C_{s}\left(\|L u\|_{s}+\sum_{i+h \leqslant s}^{*} A_{i+2}\|u\|_{j}\right),\left(u \in C_{0}^{\infty}(\Omega)\right) .
$$

By induction with respect to $s$, Lemmas $3.6,3.7$ and 3.8 follow from Lemmas 3.3 , 3.4 and 3.6.

Lemma 3.9. Assume that $L$ is uniformly elliptic in $\Omega$, that is

$$
a^{i j}(x) \xi_{i} \xi_{j} \geqslant \lambda_{0}|\xi|^{2}, \lambda_{0}=\text { constant }>0 .
$$


Then there is a constant $C^{\prime} \geqslant 0$ such that

$$
\|u\|_{1} \leqslant C\left(\lambda_{0}^{-1} A(0)\|L u\|_{0}+\lambda_{0}^{-2} A^{2}(0) A(2)\|u\|_{0}\right),\left(u \in C^{\infty}(\bar{\Omega}),\left.u\right|_{\partial \Omega}=0\right)
$$

and

$$
\|u\|_{s} \leqslant C\left(\lambda_{0}^{-s} A^{2}(0)\|L u\|_{o-1}+\lambda_{0}^{-2 s} A^{2 s}(0) \sum_{\substack{i+j \leqslant s-1 \\ j<s-1}} A(i+1)\|u\|_{j+1}\right),
$$

It is easy to prove (3.17). In fact, we have only to apply the standard techniques for the linear elliptic operator $L$ and calculate several constants exactly, and use the inequalities $A(k) \geqslant \max \left(\lambda_{0}, 1\right)$. By induction with respect to $s$ and patient calculation, (3.18) follows from (3.17).

Proof of THEOREM 3.5: Let us consider the following cut-off functions: $x \in$ $C_{0}^{\infty}, \bar{x}, \tilde{x} \in C_{0}^{\infty}(\Omega \backslash S), 0 \leqslant \chi, \bar{x}, \tilde{\bar{x}} \leqslant 1, \chi=1$ in a neighbourhood of $S$ in $U$ and $\partial_{i} \chi \subset \subset \tilde{\chi} \subset \subset \tilde{\chi}$ for any $i$. Since $\|u\|_{0} \leqslant\|(1-\chi) u\|_{s}\|\chi u\|_{s}$, it will suffice to estimate

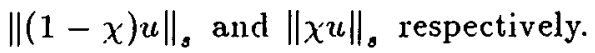

Proof OF (3.12): By (3.18) and (3.15), we have

$$
\|(1-\chi) u\|_{s} \leqslant C_{s, \lambda}\left(\|L u\|_{s-1}+\sum_{\substack{i+j \leqslant s \\ j<s}}^{*} A(i+2)\|u\|_{j}\right) .
$$

Here we note that $C_{\theta, \lambda}$ is independent of the coefficients of $L$ but depends on $\inf _{p p(1-x)} \lambda(x) \cdot(3.13)$ and $(3.16)$ give

$$
\|\chi u\|_{s} \leqslant C_{s} K\left(\|L u\|_{s}+\|[\chi, L] u\|_{0}+\sum_{\substack{i+j \leqslant s \\ j<s}}^{*} A_{i+2}\|u\|_{j}\right)+C_{s}^{\prime} K A_{2}\|u\|_{s},
$$

where $C_{x}^{\prime \prime}$ is independent of $\chi, \bar{\chi}$ and $\tilde{\tilde{\chi}}$. Since $[\chi, L] u=[\chi,(\bar{\chi} L)](\overline{\bar{\chi}} u)$ and $\bar{\chi} L(\tilde{\bar{\chi}} u)=$ $\tilde{\chi} L u,(3.15)$ shows that

$$
\|[\chi, L] u\|_{s} \leqslant C_{s}\left(\|L u\|_{s}+\sum_{\substack{i+j \leqslant s \\ j<s}}^{*} A(i+2)\|u\|_{j}+A(2)\|\tilde{\chi} u\|_{s}\right) .
$$


By (3.18), (3.15) and $A(2) \leqslant 1$, we have

$$
\|\tilde{\bar{\chi}} u\|_{s} \leqslant C_{s, \lambda}\left(\|L u\|_{s-1}+\sum_{\substack{i+j \leqslant s \\ j<s}}^{*} A(i+2)\|u\|_{j}\right),
$$

where $C_{,, \lambda}$ is independent of the coefficients of $L$ but depends on $\inf _{\operatorname{supp} \hat{x}} \lambda$. Combining (3.20) - (3.22), we obtain

$$
\|\chi u\|_{s} \leqslant C_{s, \lambda}\left(\|L u\|_{s}+\sum_{\substack{i+j \leqslant s \\ j<s}}^{*} A(i+2)\|u\|_{j}\right)+C_{s}^{\prime} K A_{2}\|u\|_{s} .
$$

Since $K A_{2} \geqslant 0$ is sufficiently small, (3.19) and (2.23) complete the proof of (3.12).

Proof of (3.11): By $(3.17),(3.15)$ and $A(2) \leqslant 1$, we have

$$
\|(1-\chi) u\|_{1} \leqslant C_{1, \lambda}^{\prime}\left(\|L u\|_{0}+\|u\|_{0}\right) .
$$

(3.13), (3.16), $[x, L] u=[x,(\tilde{x} L)](\tilde{x} u), \tilde{x} L(\tilde{\bar{x}} u)=\tilde{x} L u,(3.15)$ and $A(2) \leqslant 1$ show that

$$
\|\chi u\|_{1} \leqslant C_{1}^{\prime} I\left(\|L u\|_{1}+\|\tilde{\tilde{\chi}} u\|_{1}\right)+C_{1}^{\prime} K A_{2}\|u\|_{1}
$$

where $C_{1}^{\prime}$ is independent of $x, \bar{x}$ and $\tilde{x} \cdot(3.17),(3.15)$ and $A(2) \leqslant 1$ give

$$
\|\tilde{\tilde{\chi}} u\|_{1} \leqslant C_{1, \lambda}\left(\|L u\|_{0}+\|u\|_{0}\right) \text {. }
$$

Since $K A_{2} \geqslant 0$ is sufficiently small, by combining (3.24) - (3.26), we obtain (3.11).

Proof of (3.10): (3.4) gives

$$
\|(1-\chi) u\|_{0}^{2} \leqslant C_{0, \lambda}\|L u\|_{0}\|u\|_{0} .
$$

(3.13) implies

$$
\|\chi u\|_{0}^{2} \leqslant C_{0} K\left(\|L u\|_{0}^{2}+\|[\chi, L] u\|_{0}^{2}\right) .
$$

Since $[\chi, L] u=[x,(\bar{x} L)](\overline{\bar{x}} u), \bar{x} L(\overline{\bar{x}} u)=\bar{x} L u$ and $A(2) \leqslant 1,(3.9)$ shows that

$$
\|[x, L] u\|_{0}^{2} \leqslant C_{0}\left(\|L u\|_{0}^{2}+\|\overline{\hat{x}} u\|_{0}^{2}\right)
$$

By (3.4), we have

$$
\|\tilde{\bar{\chi}} u\|_{0}^{2} \leqslant C_{0, \lambda}^{\prime}\|L u\|_{0}\|u\|_{0} .
$$

Combining (3.27) - (3.30), we obtain (3.10). 


\section{Proof of Theorems $1.3-1.5$}

In this section, we use the notation introduced in Sections 1 and 2. According to

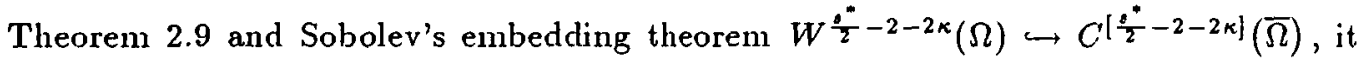
will suffice for the existence of a solution to find a good approximating solution $u_{\varepsilon}$ and a suitable elliptic operator $M$ which satisfy Assumption 2.4, 2.5 and 2.6. The uniqueness of the solution follows immediately from the maximum principle (see [1]). Cirudely speaking, Assumptions 2.4 and 2.5 are easy but Assumption 2.6 is tough. Fortunately, by virtue of Theorem 3.5 we can overcome this difficulty.

Proof of Theonem 1.3: Without loss of generality, we may assume that

$$
|g|_{4} \leqslant \frac{1}{2},\|F[g]\|_{s^{*}}>0 \text {. }
$$

In fact, since the Dirichlet problem

$$
v_{11} v_{22}-v_{12}^{2}=\left(2|g|_{4}\right)^{-2} f \quad \text { in } \quad \Omega, \quad v=\left(2|g|_{4}\right)^{-1} g \quad \text { on } \quad \partial \Omega
$$

is equivalent to (1.1), $|g|_{4} \leqslant 1 / 2$ is no actual restriction, and further, if $\|F[g]\|_{s^{*}}=0$, then the statement of Theorem 1.3 turns out to be trivial.

We put

$$
\varepsilon=\left\{\max \left(\mid F[g]\left\|_{0},\right\| F[g] \|_{g^{*}}\right)\right\}^{\frac{2}{e^{*}+2}}, \delta=1, \theta=\varepsilon^{-\frac{1}{2}}, u_{\varepsilon}=g
$$

Then it is easy to verify that Assumption 2.4 is satisfied.

By the assumption of Theorem 1.3, $g_{\nu \nu}>0$ in a neighbourhood $V$ of $S$ in $\Omega$ and $\operatorname{det}\left(g_{i j}\right)>0$ in $\Omega \backslash V$. Hence, there is a linear elliptic operator $M=\alpha^{i j} \partial_{i} \partial_{j}$ with real constant coefficients satisfying

$$
M g \geqslant 2 \text { in } \Omega
$$

By virtue of (4.3), we can construct a sequence $\left\{u_{n}\right\}$ as in Section 2 . In fact, if we already have $u_{0}, u_{1}, \ldots, u_{n}$ satisfying $(2.20)-(2.22),(2.27)-(2.28)$ and $(2.34)_{j \leqslant n}$ $(2.39)_{j \leqslant n}$, then, by $(4.2),(4.3),(2.4)$ and $(2.35)_{n}, M\left(u_{\varepsilon}+\bar{u}_{n}\right) \geqslant 2-K \sqrt{\varepsilon} ;$ this implies that Assumption 2.5 is satisfied when $\varepsilon>0$ is sufficiently small. Therefore, we can find a solution $v_{n} \in C^{\infty}(\bar{\Omega})$ of the Dirichlet problem (2.13) and obtain $u_{n+1}=u_{n}+v_{n}$.

Let us take an auxiliary function $\Phi(x)=\bar{\nu} \cdot(x-p)$, where $\bar{\nu}=\left(\nu_{1}, \bar{\nu}_{2}\right)=\left(-\nu_{2}, \nu_{1}\right)$ and $S=\{p\}$. Then Lemma 3.1 shows that

$$
\begin{gathered}
t^{2} \int\left(a_{\varepsilon_{n}}^{i j}\left[u_{\epsilon}+\bar{u}_{n}\right] \bar{\nu}_{i} \bar{\nu}_{j}\right) u^{2} d x \leqslant K \int\left|L_{e_{n}}\left[u_{\epsilon}+\bar{u}_{n}\right] u \cdot u\right| d x \\
\left(u \in C^{\infty}(\overline{U(\Phi, t)}),\left.u\right|_{O U(\Phi, t)}=0\right)
\end{gathered}
$$


for all $t \geqslant 1$, where $K=$ constant $\geqslant 0$, independent of $L_{e_{n}}\left[u_{\epsilon}+\tilde{u}_{n}\right]$ and $U(\Phi, t)$. Direct computation gives

$$
a_{\varepsilon_{n}}^{i j}\left[u_{\epsilon}+\bar{u}_{n}\right] \bar{\nu}_{i} \bar{\nu}_{j} \geqslant g_{\nu \nu}-|\nu|^{2}\left|\tilde{u}_{n}\right|_{2}-\varepsilon_{n}\left(\alpha^{i j} \bar{\nu}_{i} \bar{\nu}_{j}\right)\left|g+\tilde{u}_{n}\right|_{2} .
$$

By (2.1), (2.4), (2.35) ${ }_{n}$ and $(2.39)_{n},|\nu|^{2}\left|\bar{u}_{n}\right|_{2}+\varepsilon_{n}\left(\alpha^{i j} \bar{\nu}_{i} \nu_{j}\right)\left|g+\bar{u}_{n}\right|_{2}$ is sufficiently close to zero, while the assumption of Theorem 1.3 says that $g_{\nu \nu}$ is strictly positive. Thus, (4.4) implies that

$$
\|u\|_{0} \leqslant t^{-2} C\left\|L_{\varepsilon_{n}}\left[u_{\varepsilon}+\bar{u}_{n}\right] u\right\|_{0} \quad\left(u \in C_{0}^{\infty}(U(\Phi, t))\right)
$$

for all $t \geqslant 1$. By $(4.1),(2.35)_{n}$ and $(2.39)_{n}$, we easily have

$$
\left|a_{e_{n}}^{i j}\left[u_{\varepsilon}+\bar{u}_{n}\right]\right|_{2} \leqslant 1 \text {. }
$$

According to Theorem 3.5, (2.21) and (2.22) of Assumption 2.6 follow from (4.6) and (4.7).

Proof of Theorem 1.4: The proof of Theorem 1.4 is almost the same as that of Theorem 1.3. In fact, we have only to take an auxiliary function $\Phi(x)=\bar{\tau} \cdot(x-p)$ instead of $\bar{\nu} \cdot(x-p)$, where $\bar{\tau}=\left(-\tau_{2}, \tau_{1}\right)$ and $p$ is a fixed point on $S$.

Proof of Theorem 1.5: Without loss of generality, we may assume (4.1). We take $\varepsilon, \delta, \theta$ and $u_{\varepsilon}$ as in (4.2). Then, as in the proof of Theorem 1.3, it is easy to verify that Assumptions 2.3 and 2.4 are satisfied and also, to construct the sequence $\left\{u_{n}\right\}$ satisfying $(2.27)-(2.28)$ and $(2.34)_{j}-(2.39)_{j}$.

We take an auxiliary function $\Phi(x)=\alpha \bar{\nu} \cdot(x-p)$, where $\alpha$ is a positive constant, $\bar{\nu}=\left(\bar{\nu}_{1}, \bar{\nu}_{2}\right)=\left(-\nu_{2}, \nu_{1}\right)$ and $p$ is a fixed point on $S$. Lemma 3.1 gives

$$
\begin{gathered}
\int\left(a_{\varepsilon_{n}}^{i j}\left[u_{\varepsilon}+\tilde{u}_{n}\right] \bar{\nu}_{i} \bar{\nu}_{j}\right) u^{2} d x \leqslant K \int\left|L_{\varepsilon_{n}}\left[u_{\varepsilon}+\tilde{u}_{n}\right] u \cdot u\right| d x \\
\left(u \in C^{\infty}(\overline{U(\Phi, 1)}),\left.u\right|_{\partial U(\Phi, 1)}=0\right),
\end{gathered}
$$

where $K=$ constant $\geqslant 0$ is independent of $L_{\varepsilon_{n}}\left[u_{\varepsilon}+\tilde{u}_{n}\right]$ and $U(\Phi, 1)$. Here we take $\alpha>0$ sufficiently small so that $S \subset U(\Phi, 1)$. Direct computation shows $(4.5)$, so we have, by $(2.1),(2.4),(2.35)_{n}$ and $(2.39)_{n}$,

$$
\|u\|_{0} \leqslant C\left\|L_{e_{n}}\left[u_{\varepsilon}+\bar{u}_{n}\right] u\right\|_{0} \quad\left(u \in C_{0}^{\infty}(\Phi, 1)\right) .
$$

$0<\varepsilon<<1,\left|D^{4} g\right|_{0}<<1,(4.1),(2.35)_{n}$ and $(2.39)_{n}$ give

$$
\left|D^{2} a_{e_{n}}^{i j}\left[u_{\varepsilon}+\tilde{u}_{n}\right]\right|_{0}<<1
$$

and

$$
\left|a_{\varepsilon_{n}}^{i j}\left[u_{e}+\tilde{u}_{n}\right]\right|_{2} \leqslant 1 \text {. }
$$

By Theorem 3.5, (4.9) - (4.11) imply (2.21) and (2.22) of Assumption 2.6. 


\section{REFERENCES}

[1] K. Amano, 'Maximum principles of degenerate elliptic-parabolic operators', Indiana Univ. Math. J. $28(1979), 545-557$.

[2] K. Amano, 'The global hypoellipticity for degenerate elliptic-parabolic operators', J. Math. Soc. Japan (to appear).

[3] I.J. Bakelman, 'Generalized elliptic solutions of the Dirichlet problem for $n$-dimensional MongoAmpère equations', part 1, Proc. Sympos. Pure Math. 45 (1986), 73-102.

[4] L. Caffarelli, L. Nirenberg and J. Spruck, 'The Dirichlet problem for nonlinear second order elliptic equation I. Monge-Ampère equation', Comm. Pure Appl. Math. 37 (1984), 369-402.

[5] Y. Chell, 'Degenerate Monge-Ampère equations' (to appear).

[6] D. Gilbarg and N.S. Trudinger, Elliptic partial differential equations of second order, 2nd edition (Springer-Verlag, Berlin, Heidelberg, New York, 1983).

[7] N.V. Krylov, 'Boundedly nonhomogeneous elliptic and parabolic equations', Math. USSR-Izv. 20 (1983), 459-492.

[8] N.V. Krylov, 'Boundedly nonhomogeneous elliptic and parabolic equations in a domain', $M a t h$. USSR-Izv. 22 (1984), 67-97.

[9] N.V. Krylov, 'On degenerate nonlinear elliptic equations', Math. USSR-Sb. 48 (1984), 307-326.

[10] C'.S. Lin, 'The local isometric embedding in $R^{3}$ of 2-dimensional Riemannian manifolds with nonnegative curvature', J. Differential Geom. 21 (1985), 213-230.

[11] P.L. Lions, 'Sur les èquations de Monge-Ampère I', Manuscripta Math. 41 (1983), 1-44.

[12] P.L. Lions, 'Sux les èquations de Monge-Ampère II', Arch. Rational Mech. Anal 89 (1985), 93-122.

[13] O.A. Oleinik and E.V. Radkevich, Second order equations with nonnegative characteristic form (Amer. Math. Soc. Providence, Rhode Island and Plenum Press, 1973).

[14] J. Rauch and B.A. Taylor, 'The Dirichlet problem for the multidimensional Monge-Ampère equations', Rocky Mountain J. Math. 7 (1977), 345-364.

[15] N.S. Trudinger, 'On degenerate fully nonlinear elliptic equation in balls', Bull. Austral. Math. Soc. 35 (1987), 299-307.

[16] J.I.E. Urbas, Elliptic equations of Monge-Ampère type (Thesis, Australian National University, 1984).

Centre for Mathematical Analysis

Australian National University

G.P.O. Box 4

C:anberra, A.C!.T. 2601

Australia 